\title{
Microstructure Evolution of the Oxidized Titanium Thin Foil
}

\author{
H.-W. Zhou* and P.I. Gouma*
}

* State University of New York at Stony Brook, Dept. of Materials Science and Engineering, Stony Brook, NY 11794-2275

Oxidized titanium foils can form regular laminar structures that may find applications in nanowire templates, $\mathrm{TiO}_{2}$ photonic crystals, chemical sensors or biosensors ${ }^{[1]}$. To be the template for growing $\mathrm{TiO}_{2}$ nanowires, the periodicity of the multilayered structure should be well controlled to match the desirable diameter for the nanowire. It has been previously shown that the processing conditions, such as the temperature and the oxygen partial pressure, determine the structure and the morphology of titanium foils after oxidation ${ }^{[2]}$. However, most of the work that had been done on titanium oxidation was focused on the kinetics of the oxidation process ${ }^{[3-5]}$. Only a few authors had studied the microstructure evolution by X-ray diffraction and conventional microscopy ${ }^{[6]}$. Up to now, very few high-resolution, high magnification images about the oxidized titanium structure are available. In this study, we use a high-resolution LEO field-emission SEM as the primary means to characterize the oxidized titanium foils. The oxidation condition was changed to observe the effect of different processing parameters, such as the temperature and the moisture, etc., on the microstructure and morphology of the oxidized foils. The results show that the stratification of the oxidized foil is counteracted by the sintering of the as-stratified layers for samples oxidized in dry oxygen. Moisture is a key parameter in forming the laminar structure in oxidized titanium foils. Higher temperature will give an improved periodicity of the laminar structure than the lower temperatures in moist ambience.

\section{Reference:}

[1] P.I. Gouma et. al,, Journal of the American Ceramic Society 83 (2000) 1007

[2] P. Kofstad et. al., Acta Chemica Scandinavica 12 (1958) 239

[3] C. Oviedo, Journal of Physics - Condensed Matter 5 (1993) A153

[4] J. Stringer, Acta Metallurgica 8 (1960) 758

[5] T. Hurlen, Journal of the Institute of Metals 89 (1960) 128

[6] H.M Flower et. al., Acta Metallurgica 22 (1974) 1339 


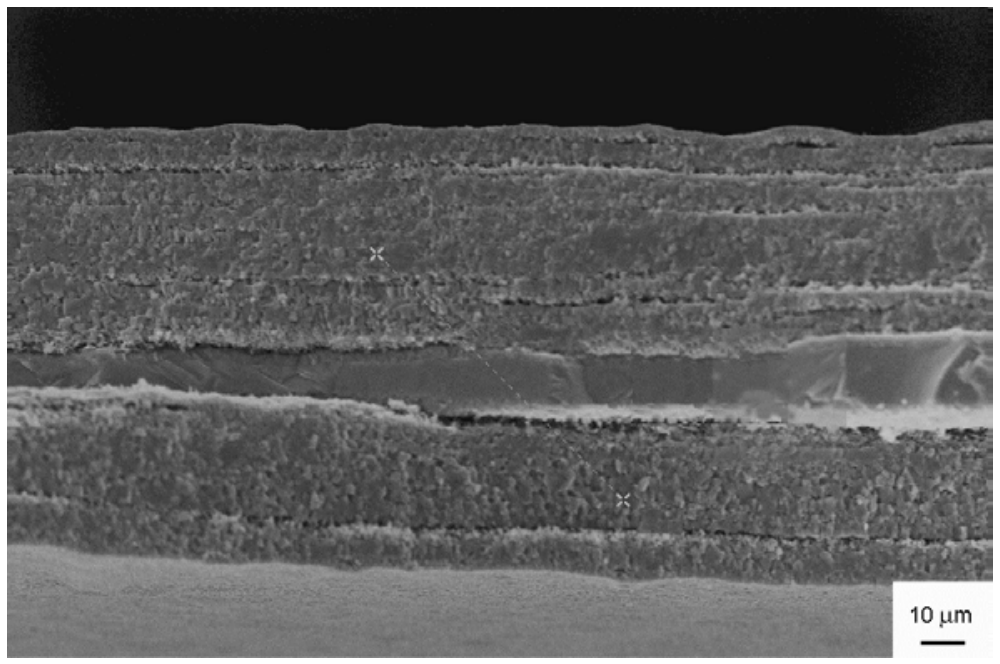

Figure 1. Cross-section of the titanium foil oxidized at $850{ }^{\circ} \mathrm{C}$ in dry oxygen for 6 hours

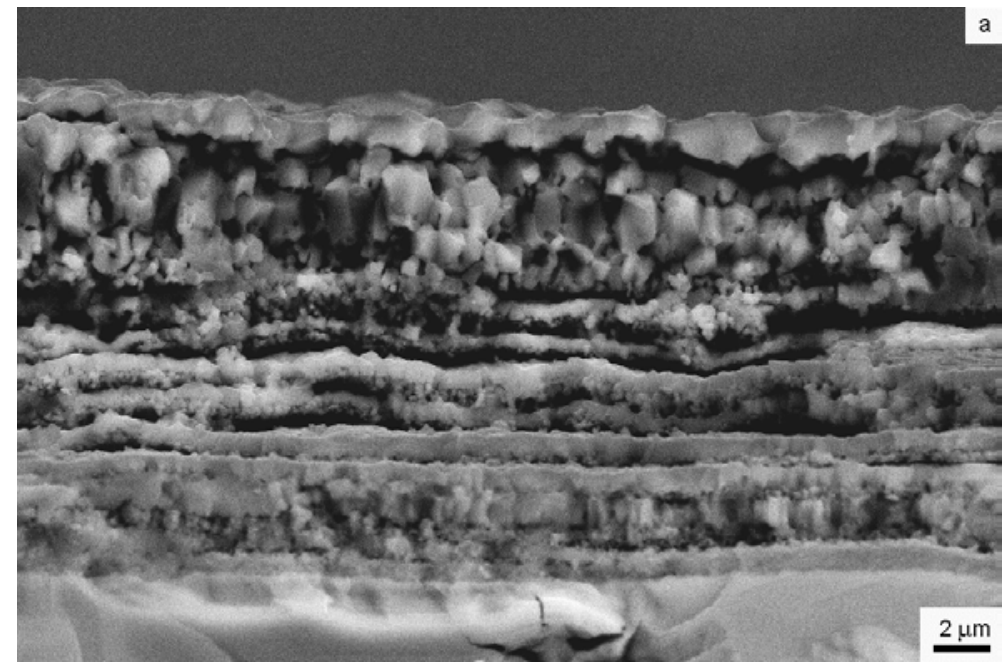

Figure 2. Cross-section images of titanium foil oxidized in moisture at $960{ }^{\circ} \mathrm{C}$ 